\title{
The Discrete Frenet Frame, Inflection Point Solitons And Curve Visualization with Applications to Folded Proteins
}

\author{
Shuangwei $\mathrm{Hu},{ }^{1,2}$ Martin Lundgren, ${ }^{1}$ and Antti J. Niemi ${ }^{1,2}$ \\ ${ }^{1}$ Department of Physics and Astronomy, Uppsala University, P.O. Box 803, S-75108, Uppsala, Sweden \\ ${ }^{2}$ Laboratoire de Mathematiques et Physique Theorique CNRS UMR 6083, \\ Fédération Denis Poisson, Université de Tours, Parc de Grandmont, F37200, Tours, France
}

(Dated: November 3, 2018)

\begin{abstract}
We develop a transfer matrix formalism to visualize the framing of discrete piecewise linear curves in three dimensional space. Our approach is based on the concept of an intrinsically discrete curve, which enables us to more effectively describe curves that in the limit where the length of line segments vanishes approach fractal structures in lieu of continuous curves. We verify that in the case of differentiable curves the continuum limit of our discrete equation does reproduce the generalized Frenet equation. As an application we consider folded proteins, their Hausdorff dimension is known to be fractal. We explain how to employ the orientation of $C_{\beta}$ carbons of amino acids along a protein backbone to introduce a preferred framing along the backbone. By analyzing the experimentally resolved fold geometries in the Protein Data Bank we observe that this $C_{\beta}$ framing relates intimately to the discrete Frenet framing. We also explain how inflection points can be located in the loops, and clarify their distinctive rôle in determining the loop structure of foldel proteins.
\end{abstract}

\section{I: INTRODUCTION}

The visualization of a three dimensional discrete framed curve is an important and widely studied topic in computer graphics, from the association of ribbons and tubes to the determination of camera gaze directions along trajectories. Potential applications range from aircraft and robot kinematics to stereo reconstruction and virtual reality [1, [2].

We are interested in addressing the problem of characterizing the physical laws that govern protein folding. For this we develop a technique for framing a general discrete and piecewise linear curve in a manner that will eventually enable us to combine the geometric problem of framing with an appropriate physical principle for frame determination. Our ultimate goal is to have an approach, where instead of purely geometric considerations the frames along a curve are determined directly from the properties of an underlying physical system. As a consequence we expect that our formalism and our results will find wide applicability well beyond the protein folding problem.

The classical theory of continuous curves in three dimensional space employs the Frenet equation [1], [2] to determine a moving coordinate frame along a sufficiently differentiable space curve. However, if the curve has inflection points and/or straight segments or if it fails to be at least three times continuously differentiable, the Frenet frame becomes either discontinuous or may not even exist. In such cases there can be good reasons to consider the option to introduce an alternative framing such as Bishop's parallel transport frame [3], a geodetic reference frame or some possibly hybrid variants [1], 2].

In this article we derive a discrete version of the Frenet equation that introduces a framing along an intrinsically discrete and piecewise linear curve in $\mathbb{R}^{3}$. We develop the general formalism for the visualization of such a curve without any underlying assumption that it approaches a continuous space curve in the limit where the maximum length of its line segments goes to zero. The continuum limit may as well be a fractal, with a nontrivial Hausdorff dimension. Thus, unlike in several approaches that we are aware of, our starting point is not in a discretization of the continuum Frenet equation. Instead our approach is intrinsically discrete, and it is based on the transfer matrix formalism that is widely used for example in lattice field theories [4. Indeed, we find it useful to adapt some notions of lattice gauge theories [4. For us this provides a valuable conceptual point of view. Moreover, since the transfer matrix formalism intrinsically incorporates self-similarity and the very concept of line segment length has no rôle in our derivations, we can effortlessly consider curves that have fractal continuum limits while at the same time ensuring that if the continuum limit exists as a class $\mathcal{C}^{3}$ space curve we recover the standard Frenet framing together with its generalized versions.

As an application we consider folded proteins, for which the continuum limit is known to be a fractal with Hausdorff dimension that is very close to three [5]. The locations of the central $C_{\alpha}$ carbon atoms along the protein determines a discrete piecewise linear curve, this is the protein backbone. We introduce a framing to the backbone by employing the $C_{\beta}$ carbon atoms of the side chain amino acids that are covalently bonded to the $C_{\alpha}$ carbons that define the backbone. The frame at the location of a given $C_{\alpha}$ carbon is determined by the directional vector that connects it with the ensuing $C_{\beta}$ carbon, together with the directional vector that connects it to the next $C_{\alpha}$ carbon along the 
backbone. By inspecting the framing of all protein structures in the Protein Data Bank (PDB) [10] we find that such a $C_{\beta}$ framing relates intimately to the discrete Frenet framing of the backbone. In particular, we conclude that for a folded protein the concept of an inflection point acquires an intrinsic biological interpretation, it coincides with the location of the center of the loop: The inflection points drive the protein loop geometry.

At an isolated inflection point of a continuous curve, the curvature which is a frame independent geometric characteristic of the curve vanishes. At such a point the Frenet frame can become discontinuous (see Figure 1). Consequently

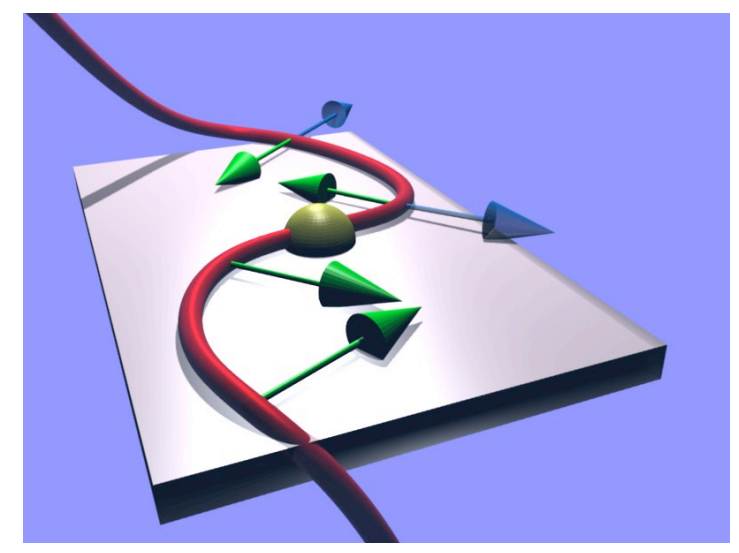

FIG. 1: A curve with inflection point (yellow ball). At each point the direction of the (Frenet frame) normal vectors (green) is towards the center of an oscullating circle. There is a discontinuity in the direction of the normal vectors when we traverse the inflection point. At this point the radius of the oscullating circle diverges and the normal vector $\mathbf{n}$ becomes abruptly reflected in the oscullating plane from one side to the other side of the curve. The blue vector equals opposite of the (reflected) normal vector $\mathbf{n}$ (see also Figure 6).

a single non-degenerate inflection point can not be removed by any local continuous deformation of the curve. An isolated non-degenerate inflection point can only be locally and continuously removed in the presence of another inflection point, by deforming the curve so that the inflection points annihilate each other in a saddle-node bifurcation. In particular a sole non-degenerate inflection point can be removed only by translating it away through an endpoint of the curve which involves a global deformation of the curve. This kind of stability enjoyed by an isolated inflection point under local deformations of the curve is the hallmark of a topological soliton. Indeed, let us recall the topological kink-soliton in a quartic double-well potential [9]

$$
\begin{gathered}
\ddot{y}=-\frac{d}{d s} V(s)=-\frac{d}{d s}\left[\frac{m^{2}}{2 c^{2}}\left(y^{2}-c^{2}\right)^{2}\right]=-\frac{2 m^{2}}{c^{2}} y\left(y^{2}-c^{2}\right) \\
y(s)=c \cdot \tanh \left[m\left(s-s_{0}\right)\right]
\end{gathered}
$$

It describes a trajectory that interpolates between the two minima $y= \pm c$ of the potential $V(s)$; See Figure 2. The center of the soliton is at the point $s=s_{0}$ where $y(s)$ vanishes. The influence of this center point to the global topology of the trajectory can not be removed by any kind of continuous local deformation $y(s) \rightarrow y(s)+\delta y(s)$, as the resulting curve continues to retain its characteristic global property that $y \rightarrow \pm c$ as $s \rightarrow \pm \infty$. Thus the deformed $y(s)$

necessarily vanishes at least at one point. The goal of the present paper is to explain how this signature behaviour of a topological soliton can be detected and described in the case of discrete piecewise linear curves, and in particular those curves that relate to the framing of folded proteins.

\section{II: THE GENERALIZED FRENET FRAME AND INFLECTION POINTS}

\section{A. A: The Generalized Frenet Frame}

We start by describing the continuum Frenet equation and its generalizations. Let $\mathbf{x}(s)$ be a space curve in $\mathbb{R}^{3}$. Its unit tangent vector

$$
\mathbf{t}=\frac{1}{\|\dot{\mathbf{x}}\|} \dot{\mathbf{x}} \equiv \frac{1}{\|\dot{\mathbf{x}}\|} \frac{d \mathbf{x}(s)}{d s}
$$



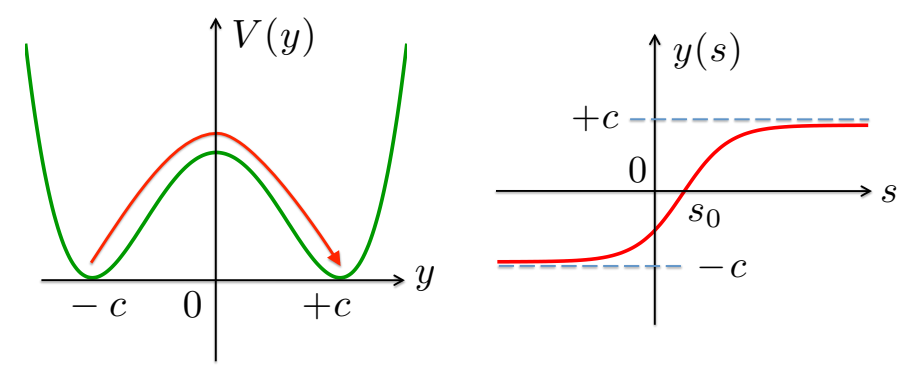

FIG. 2: The kink-soliton (right) interpolates between the two ground states at $\phi= \pm c$ of the potential (left) as $s \rightarrow \pm \infty$. It is topologically stable and can not be removed by any finite energy deformation.

(we assume that $\|\dot{\mathbf{x}}\| \neq 0$ ) is subject to the Frenet equation [1, [2]

$$
\frac{d}{d s}\left(\begin{array}{l}
\mathbf{n} \\
\mathbf{b} \\
\mathbf{t}
\end{array}\right)=\|\dot{\mathbf{x}}\|\left(\begin{array}{ccc}
0 & \tau & -\kappa \\
-\tau & 0 & 0 \\
\kappa & 0 & 0
\end{array}\right)\left(\begin{array}{l}
\mathbf{n} \\
\mathbf{b} \\
\mathbf{t}
\end{array}\right)
$$

where

$$
\mathbf{b}=\frac{\dot{\mathbf{x}} \times \ddot{\mathbf{x}}}{\|\dot{\mathbf{x}} \times \ddot{\mathbf{x}}\|}
$$

is the unit binormal vector and

$$
\mathbf{n}=\mathbf{b} \times \mathbf{t}
$$

is the unit normal vector of the curve, and

$$
\kappa(s)=\frac{\|\dot{\mathbf{x}} \times \ddot{\mathbf{x}}\|}{\|\dot{\mathbf{x}}\|^{3}}
$$

is the frame independent curvature of $\mathbf{x}(s)$ and

$$
\tau(s)=\frac{(\dot{\mathbf{x}} \times \ddot{\mathbf{x}}) \cdot \dddot{\mathbf{x}}}{\|\dot{\mathbf{x}} \times \ddot{\mathbf{x}}\|^{2}}
$$

is the torsion. The three vectors $(\mathbf{n}, \mathbf{b}, \mathbf{t})$ form the right-handed orthonormal Frenet frame at each point of the curve. In the following we shall assume with no loss of generality, that $s \in[0, L]$ measures the proper length along a curve with total length $L$ in $\mathbb{R}^{3}$ so that

$$
\|\dot{\mathbf{x}}\|=1
$$

Consider a curve with an isolated non-degenerate inflection point (or more generally a straight segment) such as the one depicted in Figure 1. At the inflection point $s=s_{0}$ the Frenet frame can not be introduced since $\kappa\left(s_{0}\right)$ vanishes; in the proper length gauge

$$
\kappa\left(s_{0}\right)=\left\|\ddot{\mathbf{x}}\left(s_{0}\right)\right\|=0
$$


Conventionally, see e.g. [6], in the presence of inflection points the Frenet equation (2) is usually introduced only piecewise between the inflection points, for those values of $s$ for which $\kappa(s)$ is nonvanishing. But there are also alternative approaches that allow for a continuous passage of the frame through the inflection point (more generally straight segments). For this we view the Frenet frame as an example of a general frame, obtained by starting from the observation that while the tangent vector $\mathbf{t}(s)$ for a given curve is unique, instead of $\{\mathbf{n}(s), \mathbf{b}(s)\}$ we may choose an arbitrary orthogonal basis $\left\{\mathbf{e}_{1}(s), \mathbf{e}_{2}(s)\right\}$ for the normal planes of the curve that are perpendicular to $\mathbf{t}(s)$, without deforming the curve. This general frame is related to the Frenet frame by a local $S O(2)$ frame rotation around the frame independent tangent vector $\mathbf{t}(s)$ (see Figure 3),

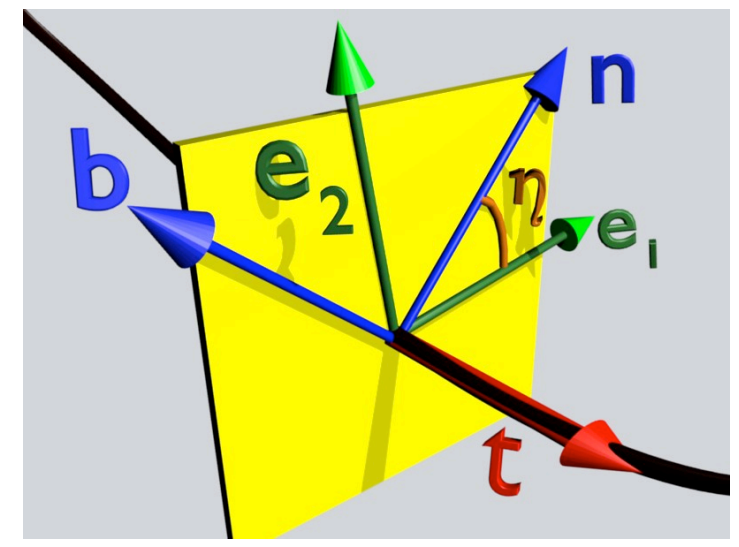

FIG. 3: The (blue) Frenet frame $(\mathbf{n}, \mathbf{b})$ and a generic (green) orthogonal frame $\left(\mathbf{e}_{1}, \mathbf{e}_{2}\right)$ on the normal plane of $\mathbf{t}$, the tangent vector of the curve.

$$
\left(\begin{array}{l}
\mathbf{n} \\
\mathbf{b}
\end{array}\right) \rightarrow\left(\begin{array}{l}
\mathbf{e}_{1} \\
\mathbf{e}_{2}
\end{array}\right)=\left(\begin{array}{cc}
\cos \eta(s) & -\sin \eta(s) \\
\sin \eta(s) & \cos \eta(s)
\end{array}\right)\left(\begin{array}{l}
\mathbf{n} \\
\mathbf{b}
\end{array}\right)
$$

The ensuing rotated version of the Frenet equation is

$$
\frac{d}{d s}\left(\begin{array}{c}
\mathbf{e}_{1} \\
\mathbf{e}_{2} \\
\mathbf{t}
\end{array}\right)=\left(\begin{array}{ccc}
0 & (\tau-\dot{\eta}) & -\kappa \cos \eta \\
-(\tau-\dot{\eta}) & 0 & -\kappa \sin \eta \\
\kappa \cos \eta & \kappa \sin \eta & 0
\end{array}\right)\left(\begin{array}{c}
\mathbf{e}_{1} \\
\mathbf{e}_{2} \\
\mathbf{t}
\end{array}\right)
$$

If we recall the adjoint basis of $S O(3)$ Lie-algebra

$$
T^{1}=\left(\begin{array}{ccc}
0 & 0 & 0 \\
0 & 0 & -1 \\
0 & 1 & 0
\end{array}\right) \quad T^{2}=\left(\begin{array}{ccc}
0 & 0 & 1 \\
0 & 0 & 0 \\
-1 & 0 & 0
\end{array}\right) \quad T^{3}=\left(\begin{array}{ccc}
0 & -1 & 0 \\
1 & 0 & 0 \\
0 & 0 & 0
\end{array}\right)
$$

where

$$
\left[T^{a}, T^{b}\right]=\epsilon^{a b c} T^{c}
$$

we find that on $\tau$ and $\kappa$ the $S O(2)$ transformation acts as follows,

$$
\begin{gathered}
\tau \rightarrow \tau-\dot{\eta} \\
\kappa T^{2} \rightarrow \kappa\left(T^{2} \cos \eta-T^{1} \sin \eta\right) \equiv e^{\eta T^{3}}\left(\kappa T^{2}\right) e^{-\eta T^{3}}
\end{gathered}
$$

If instead of $\eta \equiv 0$ that specifies the Frenet frame (Frenet gauge) we select $\eta(s)$ so that

$$
\eta(s)=\int_{0}^{s} \tau\left(s^{\prime}\right) d s^{\prime}
$$

we arrive at Bishop's parallel transport frame [3] ; 1], 2] that can be defined continuously and unambiguously through inflection points. We note that (7), (8) can be interpreted in terms of a $S O(2)$ gauge multiplet [7: The change (7) in 
$\tau(s)$ is identical to the $S O(2) \simeq U(1)$ gauge transformation of a one-dimensional gauge vector while $\kappa(s)$ transforms like a component of a $S O(2)$ scalar doublet. This leads us to a gauge invariant quantity, the complex valued Hashimoto variable [8]

$$
\xi(s)=\kappa(s) \exp \left(i \int_{0}^{s} \tau d s^{\prime}\right)
$$

When we combine (7) with a $S O(2) \subset S O(3)$ rotation $(8)$ by $\eta(s)$ around the $T^{3}$-direction of the $S O(3)$ Lie algebra, the effect on (9) can be summarized as follows,

$$
\xi(s) \rightarrow\left[\kappa(s) e^{-i \eta(s)}\right] \cdot\left[\exp \left(i \int_{0}^{s} \tau d s^{\prime}+i \eta(s)\right)\right] \cdot e^{i \eta(0)}
$$

and thus the Hasimoto variable $\xi(s)$ is manifestly independent of $\eta(s)$. (Note however, that the $\eta(0)$ dependence remains as an overall global phase ambiguity which is inherent to 10 - the local gauge invariance becomes eliminated but a global one remains.) In fact, the Hasimoto variable simply combines the two real components of the $S O(2)$ scalar doublet into a single complex valued variable, with modulus that equals the frame independent $a . k . a$. gauge invariant geometric curvature of the curve. In particular the Frenet frame is like the widely used "Unitary Gauge" in the Abelian Higgs Model [7].

We find this language of gauge transformations in connection of frame rotations introduced in [7] to be intuitively appealing and beneficial, and we shall use it frequently in the sequel.

\section{B. B: Inflection Points}

We proceed to consider a continuous curve with $n$ inflection points at $s=s_{i}$,

$$
s_{0}=0<\ldots<s_{i}<s_{i+1}<\ldots<L=s_{n+1}
$$

For simplicity we assume that the inflection points are isolated and non-degenerate zeroes of the curvature

$$
\kappa\left(s_{i}\right)=0
$$

A generalization to more involved inflection points is straightforward. We take the curve to be of class $\mathcal{C}^{3}$. This ensures that at each segment $\left(s_{i}, s_{i+1}\right)$ the curvature is of class $\mathcal{C}^{1}$. Furthermore, since the inflection points are nondegenerate, as we approach an inflection point the left and right derivatives of the curvature are non-vanishing and in the limit when $s \rightarrow s_{i}$ they become equal in magnitude but have an opposite sign,

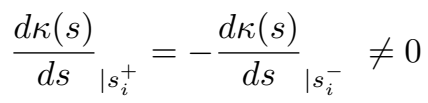

This jump in the derivative of the curvature is the signature of an inflection point in the Frenet frame. But even though the curvature $\kappa(s)$ fails to be continuously differentiable the signed curvature

$$
\tilde{\kappa}(s)=\sum_{i=0}^{n}(-1)^{i} \kappa(s) \theta\left(s-s_{i}\right) \theta\left(s_{i+1}-s\right)
$$

with $\theta(s)$ the unit step-function

$$
\theta(s)= \begin{cases}1 & s>0 \\ 0 & s<0\end{cases}
$$

is now continuously differentiable for all $s \in[0, L]$ and and in particular

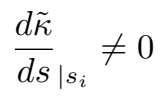

The original Frenet curvature $\kappa(s)$ and the signed curvature $\tilde{\kappa}(s)$ are related by a gauge transformation 8 of the Frenet frame, with $\eta(s)$ given by the following gauge transformation (7) of the Frenet torsion

$$
\tau(s) \rightarrow \tau(s)-\dot{\eta}(s)=\tau(s)-\pi \cdot \frac{d}{d s} \sum_{i=1}^{n-1} \theta\left(s-s_{i}\right)=\tau(s)-\pi \sum_{i=1}^{n-1} \delta\left(s-s_{i}\right)
$$


This can be immediately verified by comparing the form of (11) with that of the Hashimoto variable (9), (10). We may call this gauge transformed version of the Frenet frame the $\mathbb{Z}_{2}$-Frenet frame, its discrete version will become important to us when we consider applications to folded proteins.

For a concrete example we take the plane curve in Figure 1. For this curve, in the vicinity of the inflection point the Frenet curvature has clearly a qualitative form that may be described by the absolute value of the kink-soliton profile (1),

$$
\kappa(s) \sim \kappa_{0}\left|\tanh \left[m\left(s-s_{0}\right)\right]\right|
$$

Obviously the derivative of this curvature is discontinuous with a finite jump at the inflection point $I$ where $s=s_{0}$. This discontinuity reflects itself in the abrupt change in the direction of the (green) normal vector $\mathbf{n}$, as depicted in Figure 1. The ensuing signed curvature (11) is qualitatively described by the kink-soliton (1)

$$
\tilde{\kappa}(s) \sim \kappa_{0} \tanh \left[m\left(s-s_{0}\right)\right]
$$

and it is manifestly continuously differentiable, including the point $s=s_{0}$. Now the direction of the corresponding normal vector is also continuous through the inflection point. This is because the change in its direction becomes compensated by the change in the sign of the signed curvature when we cross the inflection point; see the blue vectors in Figure 1, and Figure 6.

\section{III: THE DISCRETE FRENET EQUATION}

\section{A. A: The Discrete Frenet Frame}

In the sequel we are primarily interested in an open and oriented, piecewise linear discrete curve that we describe by a three-vector $\mathbf{r}(s) \in \mathbb{R}^{3}$. The parameter $s \in[0, L]$ measures the arc length and $L$ is the total length of the curve. The curve is determined by its vertices $C_{i}$ that are located at the positions $\mathbf{r}_{i}=\left(\mathbf{r}_{0}, \ldots, \mathbf{r}_{n}\right)$ with $\mathbf{r}\left(s_{i}\right)=\mathbf{r}_{i}$. The endpoints of the curve are at $\mathbf{r}(0)=\mathbf{r}_{0}$ and $\mathbf{r}(L)=\mathbf{r}_{n}$. The nearest neighbor vertices $C_{i}$ and $C_{i+1}$ are connected by the line segments

$$
\mathbf{r}(s)=\frac{s-s_{i}}{s_{i+1}-s_{i}} \mathbf{r}_{i+1}-\frac{s-s_{i+1}}{s_{i+1}-s_{i}} \mathbf{r}_{i}
$$

where $s_{i}<s<s_{i+1}$. We utilize the Galilean invariance to translate the base of the curve to the origin in $\mathbb{R}^{3}$ so that

$$
\mathbf{r}_{0}=0
$$

The remaining global rotational orientation of the curve can then be fully determined by the choice of $\mathbf{r}_{1}$ and $\mathbf{r}_{2}$.

For each pair of nearest neighbor vertices $\mathbf{r}_{i+1}$ and $\mathbf{r}_{i}$ along the curve we introduce the unit tangent vector

$$
\mathbf{t}_{i}=\frac{\mathbf{r}_{i+1}-\mathbf{r}_{i}}{\left|\mathbf{r}_{i+1}-\mathbf{r}_{i}\right|}
$$

If all tangent vectors are known, the position of the $k^{\text {th }}$ vertex is given by

$$
\mathbf{r}_{k}=\sum_{i=0}^{k-1}\left|\mathbf{r}_{i+1}-\mathbf{r}_{i}\right| \cdot \mathbf{t}_{i}
$$

We now introduce the discrete Frenet frame (DF frame) at the vertex $C_{i}$ at $\mathbf{r}_{i}$. This can be done whenever the three vertices at $\mathbf{r}_{i+1}, \mathbf{r}_{i}$ and $\mathbf{r}_{i-1}$ are not located on a common line so that $\mathbf{t}_{i}$ and $\mathbf{t}_{i-1}$ are not parallel. This enables us to determine the unit binormal vector

$$
\mathbf{b}_{i}=\frac{\mathbf{t}_{i-1} \times \mathbf{t}_{i}}{\left|\mathbf{t}_{i-1} \times \mathbf{t}_{i}\right|} \quad(i=1, \ldots, n-1)
$$

and the unit normal vector

$$
\mathbf{n}_{i}=\mathbf{b}_{i} \times \mathbf{t}_{i}
$$

The orthogonal triplet $\left(\mathbf{n}_{\mathbf{i}}, \mathbf{b}_{\mathbf{i}}, \mathbf{t}_{\mathbf{i}}\right)$ constitutes the discrete Frenet frame (DF frame) for the curve at the position of the vertex $\mathbf{r}_{\mathbf{i}}$ for each $i=(1, \ldots, n-1)$, see Figure 4 . 


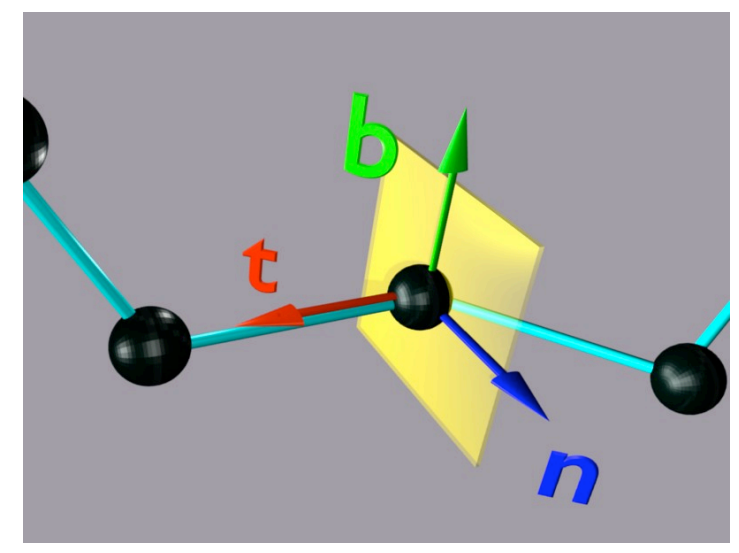

FIG. 4: A discrete piecewise linear curve is defined by its vertices $C_{i}$ and at each vertex there is an orthonormal discrete Frenet frame $\left(\mathbf{t}_{i}, \mathbf{n}_{i}, \mathbf{b}_{i}\right)$, provided $\mathbf{t}_{i-1}$ and $\mathbf{t}_{i}$ are not parallel.

\section{B. B: The Transfer Matrix}

We now proceed to derive a discretized version of the Frenet equation (DF equation) that relates the discrete Frenet frame at vertex $C_{i}$ to the discrete Frenet frame at vertex $C_{i+1}$ and allows for the construction of the curve in terms of the appropriate discrete versions of the curvature $\kappa(s)$ and torsion $\tau(s)$.

From general considerations [4] we conclude that the DF equation should involve a transfer matrix $\mathcal{R}_{i+1, i}$ that maps the DF frame at the vertex $i$ to the DF frame at the vertex $i+1$,

$$
\left(\begin{array}{c}
\mathbf{n}_{i+1} \\
\mathbf{b}_{i+1} \\
\mathbf{t}_{i+1}
\end{array}\right)=\mathcal{R}_{i+1, i}\left(\begin{array}{c}
\mathbf{n}_{i} \\
\mathbf{b}_{i} \\
\mathbf{t}_{i}
\end{array}\right)
$$

The construction of this transfer matrix then amounts to a solution of the DF equation:

$$
\left(\begin{array}{c}
\mathbf{n}_{n} \\
\mathbf{b}_{n} \\
\mathbf{t}_{n}
\end{array}\right)=\mathcal{R}_{n, n-1} \cdot \mathcal{R}_{n-1, n-2} \cdot \ldots \cdot \mathcal{R}_{2,1}\left(\begin{array}{c}
\mathbf{n}_{1} \\
\mathbf{b}_{1} \\
\mathbf{t}_{1}
\end{array}\right)
$$

so that once the transfer matrix is known for all $i=1, \ldots, n-1$, we can use 180 to construct all the Frenet frames for $i=2, \ldots, n$ and the entire curve $\mathbf{r}(s)$ using (15) together with the fact that the curve is linear in the intervals $s_{i-1}<s<s_{i}$; We recall that for the initial conditions we need to specify $\mathbf{r}_{0}$ that we have already chosen to coincide with the origin $\mathbf{r}_{0}=0$, and $\mathbf{r}_{1}$ and $\mathbf{r}_{2}$ that remove the degeneracy under global $S O(3)$ rotations of the curve in $\mathbb{R}^{3}$.

The transfer matrix $\mathcal{R}_{i+1, i}$ is an element of the adjoint representation of $S O(3)$, thus we can parametrize it in terms of Euler angles. We choose the $(z x z)$ angles

$$
\mathcal{R}_{i+1, i}=\left(\begin{array}{ccc}
-\sin \psi \sin \phi+\cos \theta \cos \psi \cos \phi & \sin \theta \cos \psi & -\sin \psi \cos \phi-\cos \theta \cos \psi \sin \phi \\
-\sin \theta \cos \phi & \cos \theta & \sin \theta \sin \phi \\
\cos \psi \sin \phi+\cos \theta \sin \psi \cos \phi & \sin \theta \sin \psi & \cos \psi \cos \phi-\cos \theta \sin \psi \sin \phi
\end{array}\right)_{i+1, i}
$$

Here the angular variables have the following ranges: For the inclination angle $\theta$ we take $\theta \in[0, \pi] \bmod (2 \pi)$ and for the two azimuthal angles we choose $\phi \in[-\pi, \pi] \bmod (2 \pi)$ and $\psi \in[-\pi, \pi] \bmod (2 \pi)$. Note that since the angular variables are elements of the transfer matrix that takes the discrete Frenet frame from the vertex $i$ to the vertex $i+1$, they are all to be interpreted as link variables that are defined on the bonds connecting the vertices.

From $(16)$ we get the following condition

$$
\mathbf{b}_{i+1} \cdot \mathbf{t}_{i}=0
$$

Thus for each bond $(i, i+1)$

$$
\sin \theta \sin \phi=0
$$


and we conclude from (14)-(17) that for all $i$ we must have

$$
\phi_{i+1, i}=0
$$

This simplifies the discrete Frenet equation into

$$
\left(\begin{array}{l}
\mathbf{n}_{i+1} \\
\mathbf{b}_{i+1} \\
\mathbf{t}_{i+1}
\end{array}\right)=\left(\begin{array}{ccc}
\cos \psi \cos \theta & \cos \psi \sin \theta & -\sin \psi \\
-\sin \theta & \cos \theta & 0 \\
\sin \psi \cos \theta & \sin \psi \sin \theta & \cos \psi
\end{array}\right)_{i+1, i} \quad\left(\begin{array}{c}
\mathbf{n}_{i} \\
\mathbf{b}_{i} \\
\mathbf{t}_{i}
\end{array}\right) \equiv \mathcal{R}_{i+1, i}\left(\begin{array}{c}
\mathbf{n}_{i} \\
\mathbf{b}_{i} \\
\mathbf{t}_{i}
\end{array}\right)
$$

Here

$$
\cos \psi_{i+1, i}=\mathbf{t}_{i+1} \cdot \mathbf{t}_{i}
$$

is the discrete bond angle and

$$
\cos \theta_{i+1, i}=\mathbf{b}_{i+1} \cdot \mathbf{b}_{i}
$$

is the discrete torsion angle. Geometrically, the bond angle $\psi_{i+1, i}$ measures the angle between $\mathbf{t}_{i+1}$ and $\mathbf{t}_{i}$ around $\mathbf{b}_{i+1}$ on the plane that is determined by the three vertices $\left(C_{i}, C_{i+1}, C_{i+2}\right)$ (Figure 5$)$. The torsion angle $\theta_{i+1, i}$ measures the angle between the two planes that are determined by the vertices $\left(C_{i-1}, C_{i}, C_{i+1}\right)$ and $\left(C_{i}, C_{i+1}, C_{i+2}\right)$, respectively (Figure 5). We give these planes an orientation in $\mathbb{R}^{3}$ by extending the range of the torsion angle from $\theta_{i+1, i} \in[0, \pi]$

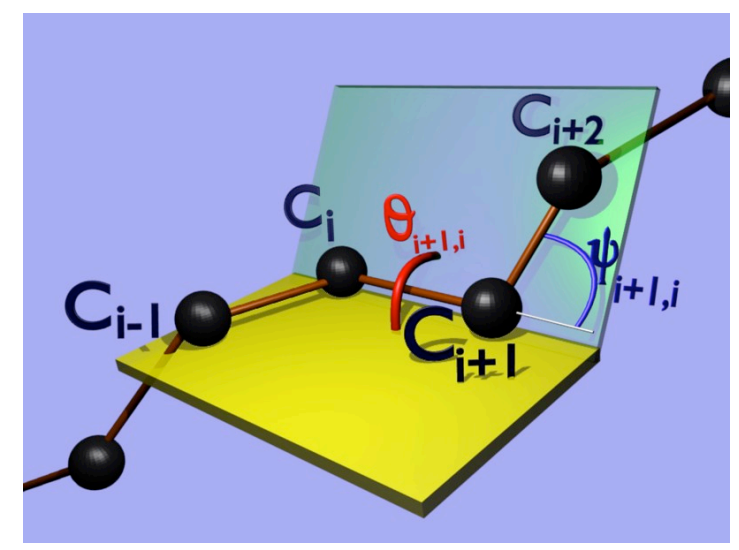

FIG. 5: The bond angle $\psi_{i+1, i}$ is determined by the three vertices $\left(C_{i-1}, C_{i}, C_{i+1}\right)$. The torsion angle $\theta_{i+1, i}$ is the angle between the two planes determined by vertices $\left(C_{i-1}, C_{i}, C_{i+1}\right)$ and $\left(C_{i}, C_{i+1}, C_{i+2}\right)$

into $\theta_{i+1, i} \in[-\pi, \pi] \bmod (2 \pi)$. This introduces a discrete $\mathbb{Z}_{2}$ symmetry

$$
\mathbb{Z}_{2}: \theta_{i+1, i} \leftrightarrow-\theta_{i+1, i}
$$

that we find useful in the sequel.

We recall the Rodrigues formula

$$
e^{\alpha \mathbb{U}}=\mathbb{I}+\mathbb{U} \sin \alpha+\mathbb{U}^{2}(1-\cos \alpha)
$$

where

$$
\mathbb{U}=\mathbf{u} \cdot \mathbf{T}=u^{a} T^{a}
$$

and $T^{a}$ are the $S O(3)$ matrices $[6]$ and $\mathbf{u}$ is a unit vector. With these we can write the transfer matrix as follows,

$$
\mathcal{R}_{i+1, i}=\exp \left\{-\psi_{i+1, i} T^{2}\right\} \cdot \exp \left\{-\theta_{i+1, i} T^{3}\right\}=\exp \{-\alpha \mathbf{v} \cdot \mathbf{T}\}_{i+1, i}
$$

where

$$
\alpha_{i+1, i}=2 \arccos \left[\frac{1}{4}\left(\mathbf{b}_{i+1} \cdot \mathbf{b}_{i}\right)\left(\mathbf{t}_{i+1} \cdot \mathbf{t}_{i}\right)\right]
$$

and

$$
\mathbf{v}_{i+1, i}=\frac{1}{\sin \frac{\alpha}{2}}\left(-\sin \frac{\psi}{2} \sin \frac{\theta}{2}, \sin \frac{\psi}{2} \cos \frac{\theta}{2}, \cos \frac{\psi}{2} \sin \frac{\theta}{2}\right)_{i+1, i}
$$




\section{C: Gauge symmetries}

Let us consider the effect of the discrete version of the local $S O(2)$ rotation (4),

$$
\left(\begin{array}{l}
\mathbf{n} \\
\mathbf{b} \\
\mathbf{t}
\end{array}\right)_{i} \rightarrow e^{\Delta_{i} T^{3}}\left(\begin{array}{l}
\mathbf{n} \\
\mathbf{b} \\
\mathbf{t}
\end{array}\right)_{i}
$$

For the covariance of the DF equation under 26) we need

$$
\begin{gathered}
e^{-\theta_{i+1, i} T^{3}} \rightarrow e^{\Delta_{i+1} T^{3}} \cdot e^{-\theta_{i+1, i} T^{3}} e^{-\Delta_{i} T^{3}} \\
e^{-\psi_{i+1, i} T^{2}} \rightarrow e^{\Delta_{i+1} T^{3}} \cdot e^{-\psi_{i+1, i} T^{2}} \cdot e^{-\Delta_{i+1} T^{3}}
\end{gathered}
$$

A direct computation shows that this implies the following transformation laws

$$
\begin{gathered}
\theta_{i+1, i} \rightarrow \theta_{i+1, i}+\Delta_{i}-\Delta_{i+1} \\
\psi_{i+1, i} T^{2} \rightarrow \psi_{i+1, i}\left(T^{2} \cos \Delta_{i+1}-T^{1} \sin \Delta_{i+1}\right)
\end{gathered}
$$

These are the discrete versions of the transformations of $\tau$ and $\kappa$ in (7), (8) respectively.

Explicitely, the gauge transformed transfer matrix is

$$
\begin{gathered}
e^{\Delta_{i+1} T^{3}} \mathcal{R}_{i+1, i} e^{-\Delta_{i} T^{3}} \equiv \mathcal{R}_{i+1, i}^{\Delta} \\
=\left(\begin{array}{ccc}
\cos \Delta \cos \theta_{\Delta} \cos \psi+\sin \Delta \sin \theta_{\Delta} & \cos \Delta \sin \theta_{\Delta} \cos \psi-\sin \Delta \cos \theta_{\Delta} & -\cos \Delta \sin \psi \\
\sin \Delta \cos \theta_{\Delta} \cos \psi-\cos \Delta \sin \theta_{\Delta} & \sin \Delta \sin \theta_{\Delta} \cos \psi+\cos \Delta \cos \theta_{\Delta} & -\sin \Delta \sin \psi \\
\cos \theta_{\Delta} \sin \psi & \sin \theta_{\Delta} \sin \psi & \cos \psi
\end{array}\right)_{i+1, i}
\end{gathered}
$$

We have here used the notation

$$
\begin{aligned}
\Delta & \Delta_{i+1} \\
\theta_{\Delta} & \equiv \theta_{i+1, i}+\Delta_{i}
\end{aligned}
$$

and the corresponding general frame Frenet equation is

$$
\left(\begin{array}{c}
\mathbf{e}_{1} \\
\mathbf{e}_{2} \\
\mathbf{t}
\end{array}\right)_{i+1}=\mathcal{R}_{i+1, i}^{\Delta}\left(\begin{array}{c}
\mathbf{e}_{1} \\
\mathbf{e}_{2} \\
\mathbf{t}
\end{array}\right)_{i}
$$

Notice that even though the explicit matrix elements in 32 do not have a manifestly covariant form in terms of the link variables, the gauge transformed transfer matrix (31) is by construction a covariant link variable.

\section{D: Continuum Limit}

The different choices of $\Delta_{i}$ in (34) correspond to different generalized Frenet frames. We shall now verify that with the general version of transfer matrix (32), this indeed yields the generalized Frenet equation (5) in the continuum limit where the distances between the vertices $C_{i}$ of the curve vanish, provided the limit is a class $\mathcal{C}^{3}$ curve.

$$
\left|\mathbf{r}_{i+1}-\mathbf{r}_{i}\right| \approx \epsilon \rightarrow 0
$$

We define

$$
\begin{array}{cl}
\psi_{i+1, i} & =\epsilon \cdot \kappa_{i+1, i} \\
\theta_{i+1, i} & =\epsilon \cdot \tau_{i+1, i} \\
\Delta_{i+1}-\Delta_{i} & =\epsilon \cdot \sigma_{i+1, i} \\
\frac{1}{2}\left(\Delta_{i+1}+\Delta_{i}\right) & =\eta_{i+1, i}
\end{array}
$$


where $\sigma_{i+i, i}$ are some finite constants. When we expand (34) in $\epsilon$ we get in the leading order

$$
\frac{1}{\epsilon}\left[\left(\begin{array}{c}
\mathbf{e}_{1} \\
\mathbf{e}_{2} \\
\mathbf{t}
\end{array}\right)_{i+1}-\left(\begin{array}{c}
\mathbf{e}_{1} \\
\mathbf{e}_{2} \\
\mathbf{t}
\end{array}\right)_{i}\right]=\left(\begin{array}{ccc}
0 & (\tau-\sigma) & -\kappa \cos \eta \\
-(\tau-\sigma) & 0 & -\kappa \sin \eta \\
\kappa \cos \eta & \kappa \sin \eta & 0
\end{array}\right)_{i+1, i}\left(\begin{array}{c}
\mathbf{e}_{1} \\
\mathbf{e}_{2} \\
\mathbf{t}
\end{array}\right)_{i}
$$

If the $\epsilon \rightarrow 0$ exists it gives us the generalized continuum Frenet equation (5), with the identification

$$
\sigma \rightarrow \dot{\eta}
$$

and the identification (35) between the discrete torsion and curvature angles with their continuum counterparts.

\section{E. E: Inflection points}

Consider a piecewise linear curve that has a single isolated inflection point located at vertex $C_{i}$; $\mathrm{A}$ generalization to several inflection points and straight segments is straightforward. By assumption, the preceding vertex $C_{i-1}$ admits a Frenet frame. Since the tangent vectors $\mathbf{t}_{i}$ and $\mathbf{t}_{i-1}$ are parallel, at the vertex $C_{i}$ both the normal vector $\mathbf{n}_{i}$ and the binormal vector $\mathbf{b}_{i}$ of a Frenet frame can not be determined and the Frenet frame at $C_{i}$ can not be introduced. Consequently the torsion angle $\theta_{i, i-1}$ can not be defined. But the definition of the bond angle involves only the tangent vectors so it can still be computed and from (21) we get

$$
\psi_{i, i-1}=0 \quad(\bmod 2 \pi)
$$

In order to introduce a framing of the curve that covers the vertex $C_{i}$, we proceed as follows: We first deform the curve slightly by moving the vertex $C_{i}$ in a direction of some arbitrarily chosen vector $\mathbf{u}$ that is not parallel with $\mathbf{t}_{i}$,

$$
\mathbf{r}_{i} \rightarrow \mathbf{r}_{i}+\epsilon \cdot \mathbf{u}
$$

Here the limit $\epsilon \rightarrow 0$ is tacitly understood. The introduction of $\mathbf{u}$ removes the inflection point from the shifted vertex $\tilde{C}_{i}$ and this enables us to introduce a $\mathbf{u}$ dependent Frenet frame at the shifted vertex $\tilde{C}_{i}$. In the limit where $\epsilon$ vanishes we get a $\mathbf{u}$ dependent frame at the original vertex $C_{i}$, obtained by transferring the Frenet frame from the vertex $C_{i-1}$ as follows,

$$
\left(\begin{array}{c}
\mathbf{e}_{1} \\
\mathbf{e}_{2} \\
\mathbf{t}
\end{array}\right)_{i}=\left(\begin{array}{ccc}
\cos \hat{\theta} & \sin \hat{\theta} & 0 \\
-\sin \hat{\theta} & \cos \hat{\theta} & 0 \\
0 & 0 & 1
\end{array}\right)_{i, i-1}\left(\begin{array}{l}
\mathbf{n} \\
\mathbf{b} \\
\mathbf{t}
\end{array}\right)_{i-1}
$$

Here $\hat{\theta}_{i, i-1}$ is now some description i.e. explicitely $\mathbf{u}$ dependent angle.

In order to establish that the frame can be chosen in a $\mathbf{u}$ independent manner we proceed to remove the explicit $\mathbf{u}$ dependence. For this we introduce the gauge transformation 290 in $(38)$ which sends

$$
\hat{\theta}_{i, i-1} \rightarrow \hat{\theta}_{i, i-1}+\Delta_{i-1}-\Delta_{i}
$$

Since we have the original Frenet frame at the vertex $C_{i-1}$, we also have

$$
\Delta_{i-1}=0
$$

But $\Delta_{i}$ is freely at our disposal and we may choose it so that any $\mathbf{u}$ dependence becomes removed. This leaves us with a $\mathbf{u}$ independent reminder that we may choose at our convenience,

$$
\hat{\theta}_{i, i-1}-\Delta_{i} \equiv \hat{\Delta}_{i, i-1}
$$

where $\hat{\Delta}_{i, i-1}$ is now by construction a $\mathbf{u}$ independent quantity, at our disposal. Different choices correspond to different gauges.

Since $\mathbf{t}_{i}$ and $\mathbf{t}_{i+1}$ are not parallel, we can proceed to construct a frame at vertex $C_{i+1}$ from the frame $\left(\mathbf{e}_{1}, \mathbf{e}_{2}, \mathbf{t}\right)_{i}$ at vertex $C_{i}$ using the transfer matrix (32). Since the remaining gauge parameters $\Delta_{k}$ with $k>i$ are all at our disposal, we may return to the Frenet frame, or select any other convenient framing, at the vertex $C_{i+1}$ and at all subsequent vertices. If the goal is to approximate a continuous space curve, in the limit of vanishing bond length the gauge parameters $\Delta_{k}$ should be selected in such a manner that in the continuum limit they yield the gauge function $\eta(s)$ and so that the ensuing discrete transfer matrix smoothly goes over to its continuum limit (36) 


\section{F. F: Discrete gauge transformations}

The transfer matrix $\mathcal{R}_{i+1, i}$ determines the curve in $\mathbb{R}^{3}$ up to rigid Galilean motions i.e. global translations and spatial rotations. The improper spatial rotation group $O(3)$ acts on each of the vertices $\mathbf{r}_{k}$ in 15 by a rotation matrix $\mathcal{O} \in O(3)$ that sends each of the $\mathbf{r}_{k}$ into

$$
\mathbf{r}_{k} \rightarrow \mathcal{O} \mathbf{r}_{k}
$$

As a consequence only the global orientation of the curve in $\mathbb{R}^{3}$ changes. An example is the improper rotation that inverts the curve in $\mathbb{R}^{3}$ by reversing the direction of each tangent vector

$$
\mathbf{t}_{i} \rightarrow-\mathbf{t}_{i}
$$

but with no effect on the $\mathbf{n}_{i}$ and $\mathbf{b}_{i}$. From the explicit form of the transfer matrix in 200 we conclude that this corresponds to the following global version of 29, , 30

$$
\begin{aligned}
& \theta_{i} \rightarrow \theta_{i} \\
& \psi_{i} \rightarrow-\psi_{i}
\end{aligned}
$$

That is, $\Delta_{i}=\pi$ for all $i$. Consequently if we include this improper rotation in our gauge structure we can restrict the range of $\psi_{i}$ from $\psi_{i} \in[-\pi, \pi] \bmod (2 \pi)$ to $\psi_{i} \in[0, \pi] \bmod (2 \pi)$, but we prefer to continue with the extended range.

Similarly, we can introduce the improper rotation that sends

$$
\mathbf{b}_{i} \rightarrow-\mathbf{b}_{i}
$$

with no effect on $\mathbf{t}_{i}$ and $\mathbf{n}_{i}$. Since the $\mathbf{t}_{i}$ remain intact the curve does not change, and from the DF equation $(20)$ we conclude that this corresponds to the following global $\mathbb{Z}_{2}$ transformation

$$
\begin{aligned}
& \theta_{i} \rightarrow-\theta_{i} \\
& \psi_{i} \rightarrow \psi_{i}
\end{aligned}
$$

This is the $\mathbb{Z}_{2}$ symmetry that we have introduced in (23), to extend the range of $\theta_{i}$ from $\theta_{i} \in[0, \pi]$ to $\theta_{i} \in[-\pi, \pi] \bmod (2 \pi)$. We note that this symmetry of the underlying curve can not be reproduced by the gauge transformation (29), 30], nevertheless the curve remains intact since the $\mathbf{t}_{i}$ do not change.

Another useful discrete transformation in our subsequent discrete curve analysis is the proper rotation that at a given vertex $C_{i}$ sends

$$
\begin{aligned}
& \mathbf{b}_{i} \rightarrow-\mathbf{b}_{i} \\
& \mathbf{n}_{i} \rightarrow-\mathbf{n}_{i}
\end{aligned}
$$

but with no effect on $\mathbf{t}_{i}$ so that the curve remains intact. This rotation is obtained by selecting $\Delta_{i+1}=\pi$ and with all $\Delta_{k}=0$ at the preceding vertices $C_{k}$ (with $k \leq i$ ). Since the $\Delta_{i+1}$ appears in the gauge transformation law of both $\theta_{i+1, i}$ and $\theta_{i+2, i+1}$, this leads to the following realization of the gauge transformation $(29),(30)$

$$
\begin{array}{ccc}
\theta_{i+1, i} & \rightarrow \theta_{i+1, i}-\pi \\
\theta_{i+2, i+1} & \rightarrow \theta_{i+1, i}+\pi \\
\psi_{i+1, i} & \rightarrow-\psi_{i+1, i}
\end{array}
$$

If we generalize this gauge transformation by selecting

$$
\Delta_{k}=\pi \quad \text { for } k \geq i+1
$$

with

$$
\Delta_{k}=0 \quad \text { for } k<i+1
$$

where the vertex $C_{i}$ is preselected, the gauge transformation becomes

$$
\begin{array}{ll}
\theta_{i+1, i} & \rightarrow \theta_{i+1, i}-\pi \\
\psi_{k+1, k} & \rightarrow-\psi_{k+1, k}
\end{array} \quad \text { for all } k \geq i
$$

Since the bond angle is the discrete version of the Frenet curvature $(35)$, we recognize here the discrete analog of the continuum gauge transformation (11), (12). For a piecewise linear discretization of a plane curve such as the one Figure 1, this enables us to introduce a framing that captures the kink-soliton behaviour (1), (13) of the inflection point, with the change of sign in curvature at the soliton position (Figure 6). 


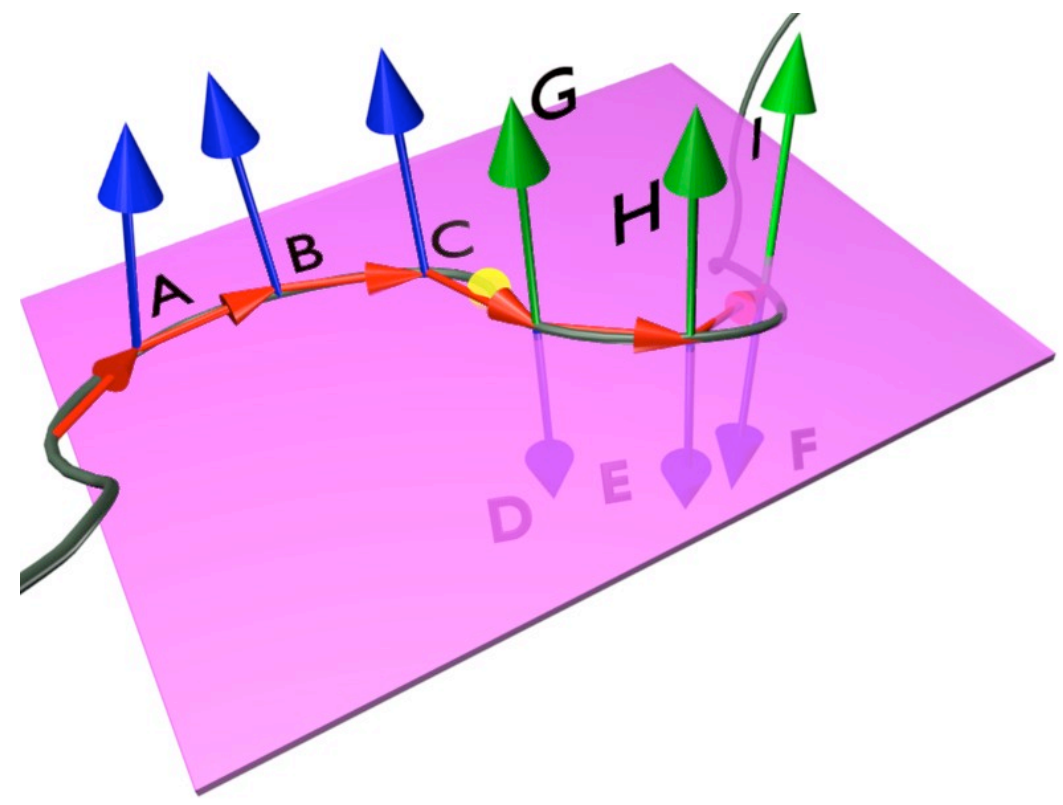

FIG. 6: A continuous plane curve with an inflection point (yellow dot) such as the one in Figure 1, together with its discrete approximation. The tangent vectors $\mathbf{t}_{i}$ (red) of the discrete approximation can be chosen so that two neighbors are never parallel and thus a discrete Frenet frame can be introduced at each vertex. When we pass through the inflection point the direction of the binormal vectors following $(\mathbf{A}, \mathbf{B}, \mathbf{C})$ becomes reflected in the plane into $(\mathbf{D}, \mathbf{E}, \mathbf{F})$ and there is a discontinuity in the Frenet framing. But if we introduce the gauge transformation $\sqrt{39}$ at vertices after the inflection point, the ensuing framing (A, B, C, G, H, I) is continuous.

\section{G. G: Curve Construction}

An example of problems where the present formalism can be applied is the construction of a discrete and piecewise linear curve from the known values of its bond and torsion angles. These angles can be constructed for example using an energy principle to locate a minimum energy configuration of some energy functional

$$
E\left(\psi_{k+1, k}, \theta_{k+1, k}\right)
$$

We may define the angles using the Frenet frame. Examples of energy functionals have been discussed in [7, [5.

Three vertices are needed to specify the position and the overall rotational orientation of the curve. To compute a single bond angle from the curve, we need three vertices while for the torsion angle we need four; See Figure 5. Consequently from the first three initial positions of the curve, $\left(\mathbf{r}_{0}, \mathbf{r}_{1}, \mathbf{r}_{2}\right)$, we can compute the first bond angle $\psi_{1,0}$. But in order to compute the first pair $\left(\psi_{2,1}, \theta_{2,1}\right)$ we also need to specify $\mathbf{r}_{3}$.

Here we are interested in the inverse problem where the set of angles $\left\{\psi_{k+1, k}, \theta_{k+1, k}\right\}$ are assumed to be known. Depending on the boundary conditions for the energy functional, the known initial data may also include numerical values of $\left(\psi_{1,0}, \theta_{1,0}\right)$, even though $\theta_{1,0}$ lacks a geometric interpretation. In such a case we can immediately proceed to the computation of the entire curve using (20) or alternatively using the transfer matrix (34), starting from an initial choice of frame $\left(\mathbf{n}_{0}, \mathbf{b}_{0}, \mathbf{t}_{0}\right)$. Different initial choices are related to each other by global i.e. index $i$ independent parameter $\Delta$ in $(29),(30)$.

We get both the frame at the vertex $k$ and its location $\mathbf{r}_{k}$ when we also employ (15), starting from a given initial value $\mathbf{r}_{0}(=0)$.

In general we expect to have a situation where the three first points $\left(\mathbf{r}_{0}, \mathbf{r}_{1}, \mathbf{r}_{2}\right)$ are given. From these points we get the two tangent vectors $\mathbf{t}_{0}$ and $\mathbf{t}_{1}$. We then use (16), (17) to complete the Frenet frame at the location $\mathbf{r}_{1}$. We identify the bond angle $\psi_{1,0}$ with the angle between the two vectors $\mathbf{t}_{0}$ and $\mathbf{t}_{1}$ using (21). This bond angle may or may not be determined by the energy functional. If it is determined, the angle between $\mathbf{t}_{0}$ and $\mathbf{t}_{1}$ is determined and instead of fully specifying $\mathbf{r}_{2}$ we only need to specify its distance from $\mathbf{r}_{1}$ and the remaining directional angle that we may call $\theta_{1,0}$. 
For a practical algorithmic implementation the following choice can be convenient,

$$
\begin{gathered}
\mathbf{r}_{0}=\delta_{1,0}\left(\begin{array}{c}
-\cos \psi_{1,0} \\
\sin \psi_{1,0} \\
0
\end{array}\right) \\
\mathbf{r}_{1}=\left(\begin{array}{l}
0 \\
0 \\
0
\end{array}\right) \\
\mathbf{t}_{0}=\left(\begin{array}{c}
\cos \psi_{1,0} \\
-\sin \psi_{1,0} \\
0
\end{array}\right) \\
\mathbf{n}_{1}=\left(\begin{array}{l}
1 \\
0 \\
0
\end{array}\right) \quad \mathbf{b}_{1}=\left(\begin{array}{l}
0 \\
1 \\
0
\end{array}\right) \quad \mathbf{t}_{1}=\left(\begin{array}{l}
0 \\
0 \\
1
\end{array}\right)
\end{gathered}
$$

where we have introduced the notation

$$
\delta_{k+1, k}=\left|\mathbf{r}_{k+1}-\mathbf{r}_{k}\right|
$$

for the segment lengths. The generalized Frenet frame together with the corresponding location of the vertex $\mathbf{r}_{i+1}$ can then be computed by iterative application of

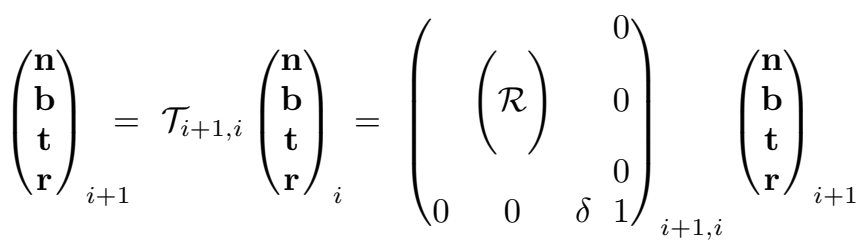

This can be directly generalized into

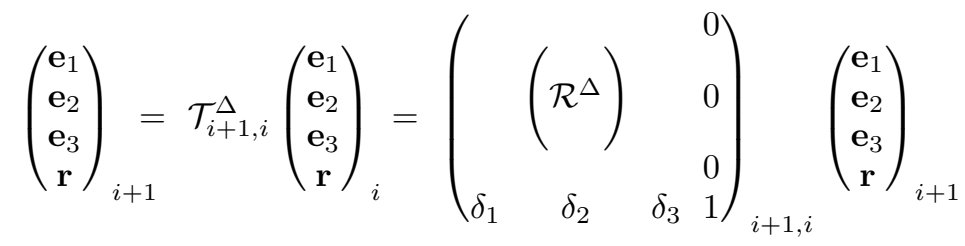

where $\mathcal{R}^{\Delta}$ is the matrix 32 and the $\delta_{1}, \delta_{2}, \delta_{3}$ are the components of the vector

$$
\vec{\delta}_{k+1, k}=\delta_{k+1, k} \cdot\left(\begin{array}{c}
\cos \alpha \sin \beta \\
\sin \alpha \sin \beta \\
\cos \beta
\end{array}\right)_{k+1, k}
$$

When $\beta=0$ (and $\Delta=0$ ) we obtain the transfer matrix (41) with $\mathbf{t}_{k}$ the tangent vector of the curve, while for general $(\alpha, \beta)$ the tangent of the curve is in the direction of $\vec{\delta}$ in the $\left(\mathbf{e}_{1}, \mathbf{e}_{2}, \mathbf{e}_{3}\right)$ frame. Thus this transfer matrix provides a rule for transporting an a priori arbitrarily oriented orthogonal frame along the curve.

Of particular interest is the construction of a discrete version of Bishop's parallel transport frame [3], as a gauge transformed version of the discrete Frenet frame. Since the Frenet frame starts with $\left(\psi_{2,1}, \theta_{2,1}\right)$ and can be constructed once $\left(\mathbf{r}_{0}, \mathbf{r}_{1}, \mathbf{r}_{2}, \mathbf{r}_{3}\right)$ are known (unless we introduce $\theta_{1,0}$ which lacks a geometric interpretation), we assume this to be the case. The discrete version of Bishop's frame is obtained by gauge transformation from the Frenet frame, by demanding that

$$
\theta_{2,1} \rightarrow \theta_{2,1}+\Delta_{1}-\Delta_{2}=0
$$


We can freely choose

$$
\Delta_{1}=0
$$

as an initial condition, and consequently we arrive at Bishop's frame by selecting

$$
\Delta_{2}=\theta_{2,1}
$$

For $\Delta_{3}$ we get similarly from

$$
\theta_{3,2} \rightarrow \theta_{3,2}+\Delta_{2}-\Delta_{3}=0
$$

that

$$
\Delta_{3}=\theta_{2,1}+\theta_{3,2}
$$

and thus the discrete version of Bishop's parallel transport frame is related to the discrete Frenet frame by gauge transformations

$$
\Delta_{k}=\sum_{i=1}^{k-1} \theta_{i+1, i}
$$

When we substitute this in 32 with 33 , we find that the transfer matrix 32 simplifies into

$$
\mathcal{R}_{i+1, i}^{B}=\left(\begin{array}{ccc}
1+\cos ^{2} \Theta_{\Delta}(\cos \psi-1) & \sin \Theta_{\Delta} \cos \Theta_{\Delta}(\cos \psi-1) & -\cos \Theta_{\Delta} \sin \psi \\
\sin \Theta_{\Delta} \cos \Theta_{\Delta}(\cos \psi-1) & 1+\sin ^{2} \Theta_{\Delta}(\cos \psi-1) & -\sin \Theta_{\Delta} \sin \psi \\
\cos \Theta_{\Delta} \sin \psi & \sin \Theta_{\Delta} \sin \psi & \cos \psi
\end{array}\right)_{i+1, i}
$$

where now

$$
\Theta_{\Delta} \equiv \sum_{k=1}^{i} \theta_{k+1, k}
$$

and with (34), we can construct the discrete version of Bishop's parallel transport frame at each vertex $C_{i}$.

\section{IV: FRAMING OF FOLDED PROTEINS}

As an application we utilize the DF equation to investigate the framing of the folded proteins in the Protein Data Bank (PDB) [10. We are particularly interested in the existence and characterization of a preferred framing that derives and directly reflects the physical properties of the folded proteins. The identification of such a preferred framing, if it exists, should help to pinpoint the physical principles that determine how proteins fold.

From the PDB we get the three dimensional coordinates of all the different atoms in a folded protein. The overall fold geometry is described by the location of the central $C_{\alpha}$ carbons that determine the protein backbone. We take the $C_{\alpha}$ carbons to be the vertices in a discrete and piecewise linear curve that models the backbone. We then use the $C_{\alpha}$ coordinates to compute the corresponding Frenet framing. For this we first apply (14), (16), (17) to obtain the orthonormal basis vectors at each vertex. We then construct the transfer matrices by evaluating the bond and torsion angles from (21) and 222.

\section{A. A: $\mathbb{Z}_{2}$ Frenet framing and solitons}

We start by analyzing in detail an explicit example, the chicken villin headpiece subdomain HP35 (PDB code 1YRF [10]). This is a naturally existing 35-residue protein, with three $\alpha$-helices separated from each other by two loops. This protein continues to be the subject to very extensive studies both experimentally [11]-[14] and theoretically [15]-[19]. We note that the overall resolution in the experimental x-ray data in PDB is 1.07 $\dot{\mathrm{A}}$ in RMSD [13.

We first compute the backbone Frenet frame bond and torsion angles $\left(\psi_{i+1, i}, \theta_{i+1, i}\right)$ from the PDB coordinates of the HP35 $C_{\alpha}$ carbons. The result is shown in Figure 7 (left).

We inquire whether the loop regions contain inflection points. As we have previously explained for example in connection of Figure 6, the inflection points can be difficult to identify in terms of the bond angles of the discrete 

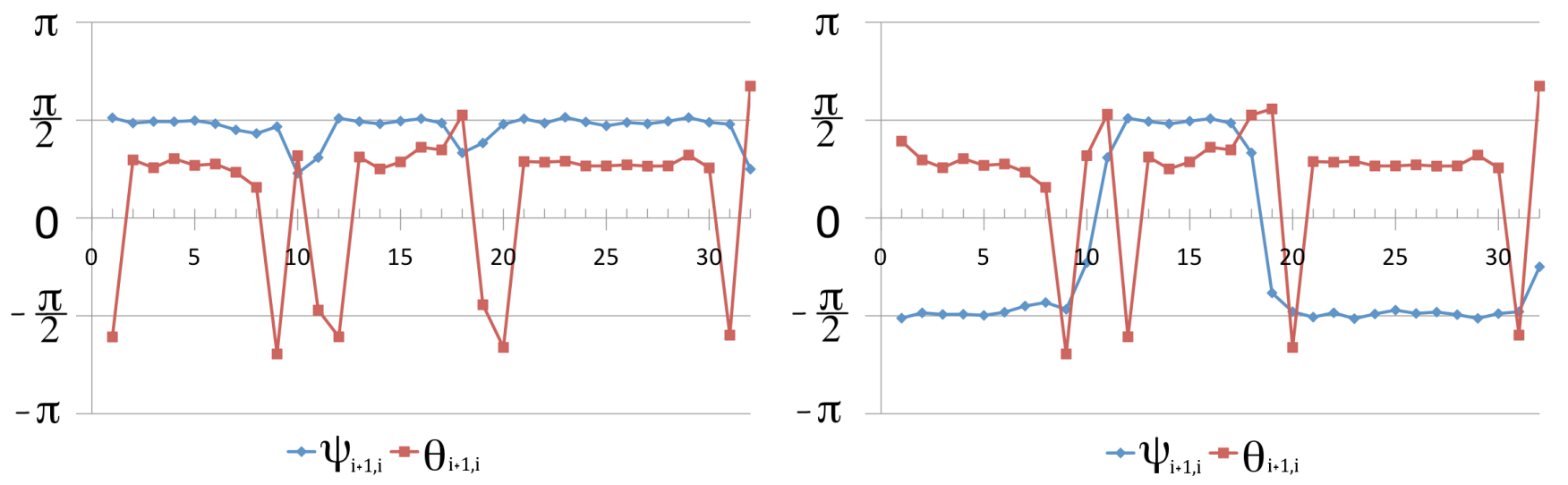

FIG. 7: Left: The Frenet frame bond angle (blue) and torsion angle (red) along the HP35 backbone. In this frame the potential presence of an inflection point is only visible in large local variations of torsion angle. Right: The outcome of $\mathbb{Z}_{2}$ gauge transformations (39) at the loop regions. The result clearly reveals the presence of inflection points, they are located between the sites where the (gauge transformed) bond angle changes its sign. This can also be used to dentify the center of the loop. Note how closely the profile of the bond angle in the right hand side picture resembles that of the kink-soliton in the r.h.s. of Figure 2 .

Frenet framing alone. But as apparent from Figure 6, we can expect that an inflection point is located in the vicinity of vertices where the Frenet frame torsion angle is subject to strong local fluctuations. Thus we proceed to inspect the data in Figure 7 (left) using the gauge transformation (39) to scrutinize the loop regions where the Frenet torsion angle in Figure 7 (left) is strongly fluctuating. This leads us to a particular version of the $\mathbb{Z}_{2}$ Frenet frame, with bond and torsion angles as in Figure 7 (right).

By comparing the bond angles in Figure 7 (right) with the kink-soliton profile in the right hand side of Figure 2 we observe that the bond angles of our gauge transformed frames at each of the loops have assumed the distinctive hallmark profile of a (discrete) kink-soliton that interpolates between the adjacent $\alpha$-helices. In particular, we can unambiguously pinpoint the centers of the loops to the locations of the inflection points on the curve: The inflection points are between the vertices where the bond angle in our gauge transformed frame changes its sign.

We have performed a similar analysis to several proteins in the PDB, and some of our results where the techniques of the present article are utilized have been reported in 20, 21, 22. The results are remarkably consistent: In every secondary superstructure that we have studied where a loop connects two $\alpha$-helices and/or $\beta$-strands, after appropriate $\mathbb{Z}_{2}$ gauge transformations the profile of the bond angles in the loop can be described with sub-Ängström accuracy in terms of a discrete version of the kink-soliton in Figure 2. The two asymptotic ground states at $s= \pm c$ in this Figure correspond to the $\alpha$-helices and/or $\beta$-strands at the ends of the loop. For the $\alpha$-helices we have the Frenet frame values very close to

$$
(\psi, \theta)_{\alpha} \approx(1.57,0.87) \sim\left(\frac{\pi}{2}, 1\right)
$$

The $\beta$ strands can also be interpreted as helices, but in the "collapsed" limit with the approximative values

$$
(\psi, \theta)_{\beta} \approx( \pm 1.0,-2.9) \sim( \pm 1,-\pi)
$$

Consequently it appears that these $\alpha$-helix/ $\beta$-strand - loop - $\alpha$-helix/ $\beta$-strand superstructures are indeed inflection point solitons with the qualitative profile of (1). We remark that a long loop may also consist of a number of inflection points i.e. it can be a multi-soliton configuration.

\section{B. B: Physics based framing}

In every amino acid except glycine, there is a $C_{\beta}$ carbon that is covalently bonded to a $C_{\alpha}$ carbon. The positioning of these $C_{\beta}$ carbons in relation to their $C_{\alpha}$ carbons characterizes the relative orientation of the amino acid side chains along the protein backbone, and can be used to introduce a distinctive framing of the backbone; the case of glycine can be treated like that of an inflection point. Since the interactions between different amino acids are presumed to have a pivotal rôle both during the folding process and in the stabilization of the native fold, the $C_{\beta}$ framing should 
be a natural choice to intimately reflect the physical principles that determine the fold geometry of the backbone. Consequently one way to try and understand the physical principles that determine how a protein folds, could be to investigate the $C_{\beta}$ framing along the protein backbone. Here we propose that a practical approach is to look for gauge parameters 26 that relate the $C_{\beta}$ frames to some purely geometrically determined frames such as the Frenet frames or parallel transport frames. The identification of the rules that determine the relevant gauge parameters $\Delta_{i}$ should then provide insight to the physical principles that underlie the protein folding phenomenon.

The $C_{\beta}$ framing is constructed from the tangent vectors $\mathbf{t}$ of the backbone and the unit vectors $\mathbf{c}$ that point from the $C_{\alpha}$ carbons towards their $C_{\beta}$ carbon. The framing is obtained by Gram-Schmidt algorithm, by first introducing the unit vector

$$
\mathbf{p}=\frac{\mathbf{t} \times \mathbf{c}}{\|\mathbf{t} \times \mathbf{c}\|}
$$

and then completing it into an orthonormal frame $(\mathbf{t}, \mathbf{p}, \mathbf{q})$ at each $C_{\alpha}$ vertex, where

$$
\mathbf{q}=\mathbf{t} \times \mathbf{p}
$$

In order to characterize the rules that determine the gauge parameter $\Delta_{i}$ relating a $C_{\beta}$ frame to the corresponding Frenet frame, we have investigated the statistical distribution of the $\mathbf{c}_{i}$ vectors in the PDB proteins in the Frenet framing of the backbone. For this we introduce, at each backbone vertex, the inclination angle $\chi_{i} \in[0, \pi]$ between the tangent vector $\mathbf{t}_{i}$ and the corresponding vector $\mathbf{c}_{i}$, together with the azimuthal angle $\varphi_{i} \in[-\pi, \pi]$ between the normal vector $\mathbf{n}_{i}$ and the projection of $\mathbf{c}_{i}$ on the $\left(\mathbf{n}_{i}, \mathbf{b}_{i}\right)$ plane; see Figure 8.

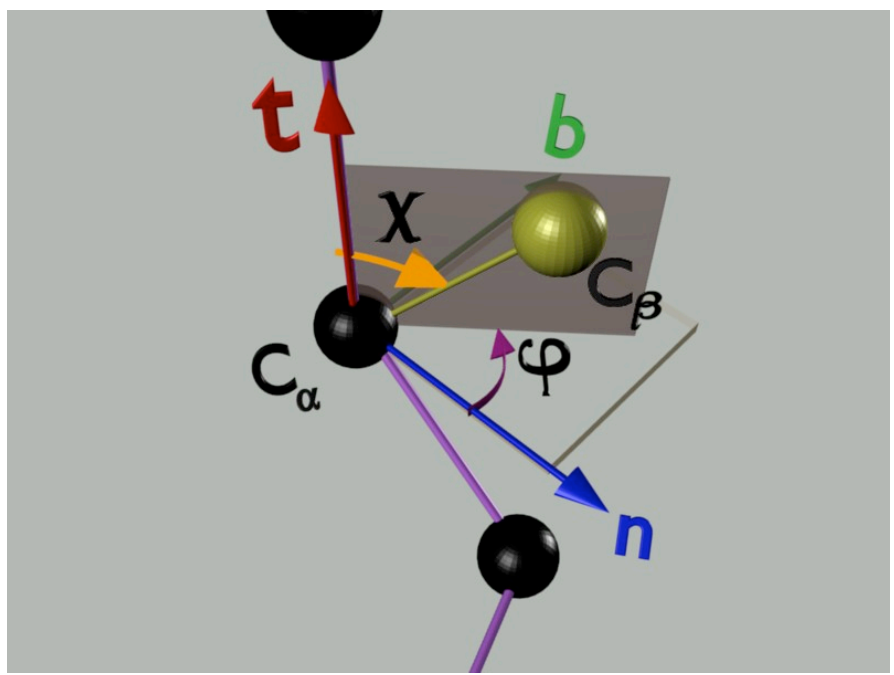

FIG. 8: The definition of the angles $\chi_{i}$ and $\varphi_{i}$ that describe the location of the $i^{\text {th }} C_{\beta}$ carbon with respect to the Frenet frame along the $C_{\alpha}$ backbone. The distance between the $C_{\alpha}$ and $C_{\beta}$ carbons is within the range of 1.56-1.57 $\dot{\mathrm{A}}$.

We first consider the $C_{\beta}$ framing of the HP35. When we compute the directions of the individual vectors $\mathbf{c}_{i}$ in the Frenet frame, we get the result that we display in Figure 9. Remarkably, the directions of the $\mathbf{c}_{i}$ vectors in the Frenet frame are relatively site independent. This implies that at least in the case of HP35, the parameters $\Delta_{i}$ that relate the $C_{\beta}$ frame to the Frenet frame can be assigned to a high accuracy a constant and site independent value: The physically determined orthonormalized $C_{\beta}$ frame appears to differ from the purely geometrically determined Frenet frame only by small nutations in the direction of the vectors $\mathbf{c}$ in the Frenet frame. We observe that these nutations are somewhat smaller in the helix regions that in the loops.

We conclude that since the Frenet framing of HP35 is determined entirely by the backbone geometry so are the orientations of the amino acids, with a surprisingly good accuracy.

In the general case, we have inspected the correlation between the $C_{\beta}$ framing and the Frenet framing by performing a statistical analysis of the directional distribution of the $\mathbf{c}$-vectors in the backbone Frenet frames for all amino acids in PDB. Our results are summarized by Figure 10 where we display the statistical distribution of the angles $\left(\varphi_{i}, \chi_{i}\right)$ that we have defined in Figure 8. We have used the PDB definition to identify the three structures we display separately $(\alpha$-helix, $\beta$-strand, loop) but we note that there are sometimes ambiguities in determining whether a particular amino 


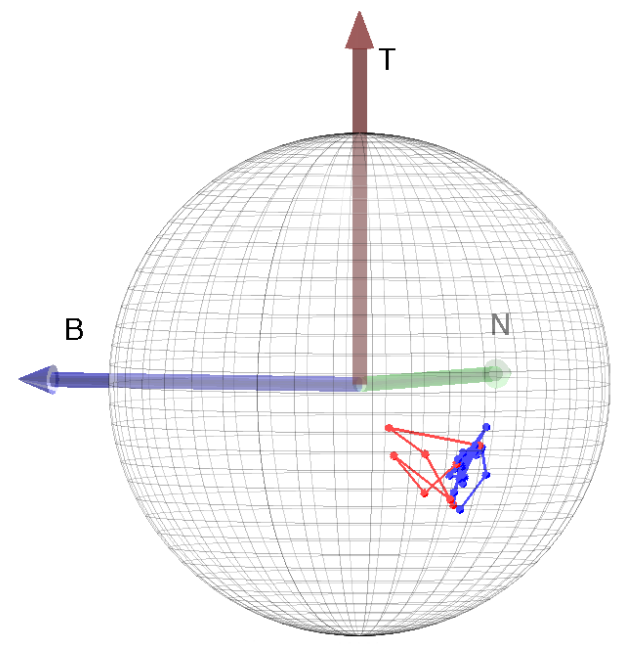

FIG. 9: The nutation in the direction of the vectors $\mathbf{c}_{i}$ in the Frenet frame along 1YRF backbone. The blue dots are the $C_{\alpha}$ carbons in the helices, and the red dots are the $C_{\alpha}$ carbons that are located in the loops.
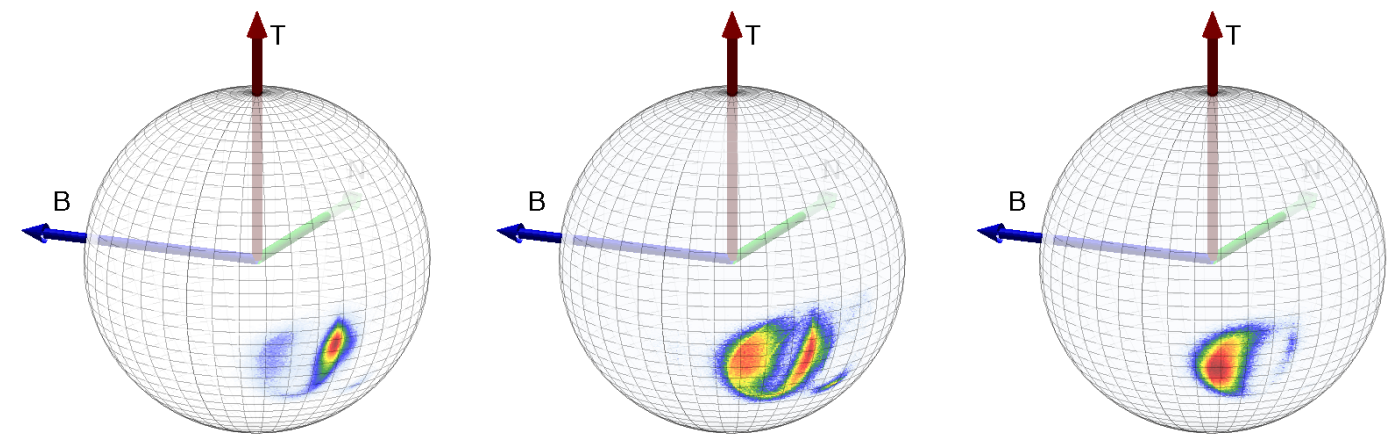

FIG. 10: Kent plots of the $C_{\beta}$ carbon vectors $\mathbf{c}$ for all sites of all proteins presently in PDB, with color intensity proportional to the number of vectors. For $\alpha$-helices (left), the direction of $\mathbf{c}$ nutates very little around the direction $(\chi, \varphi) \approx(1.84,-2.20)$. For $\beta$-strands (right) the nutation is somewhat more spread, but still very clearly concentrated around $(\chi, \varphi) \approx(1.96,-2.47)$. Finally, for loops (center) we observe the formation of a narrow arc that connects the $\alpha$ and $\beta$ directions.

acid belongs to a $\alpha$-helix, $\beta$-strand or a loop in particular when the amino acid is located in the vicinity of the border between these three classes.

We find that the observation we have made in the case of HP35 persists: The orientations of the $C_{\beta}$ carbons in the Frenet frames are quite inert and essentially protein and amino acid independent. There is only a slight nutation around the statistical average value. Furthermore, the directions for the $\alpha$-helices and $\beta$-strands are also almost the same, the difference in the statistical average is surprisingly small but nevertheless noticeable. In the case of loops, we find that the statistical distribution of the vector $\mathbf{c}$ in the Frenet frame displays a thin band that connects the $\alpha$-helices and $\beta$-strands. This universality is somewhat unexpected, since only a small proportion of the loops connect an $\alpha$-helix with a $\beta$-strand.

The overlapping regions between the three different classes in the Kent plots of Figure 10 can be at least partly explained by the uncertainty in classifying amino acids in the vicinity of the border regions. We expect that a careful scrutiny of the class assignments of these amino acids will sharpen our statistical results. Alternatively, our approach 
could be developed into a technique to determine a more definite classification of those amino acids that are located in the border regions separating the $\alpha$-helices, $\beta$-strands and the loops from each other. But even at this level of classifying the amino acids the results of our analysis imply that almost independently of the protein, when we traverse its backbone by orienting the camera gaze direction so that it remains fixed in the Frenet frames, the directions of the $C_{\beta}$ carbons are subject to only small nutations.

In Figure 11 we display the histograms for the components of the $C_{\beta}$ vectors $\mathbf{c}_{i}$ in terms of the $\chi$ and $\varphi$ angles defined in Figure 8. These histograms confirm that the directional variations of the $\mathbf{c}_{i}$ are surprisingly inert.

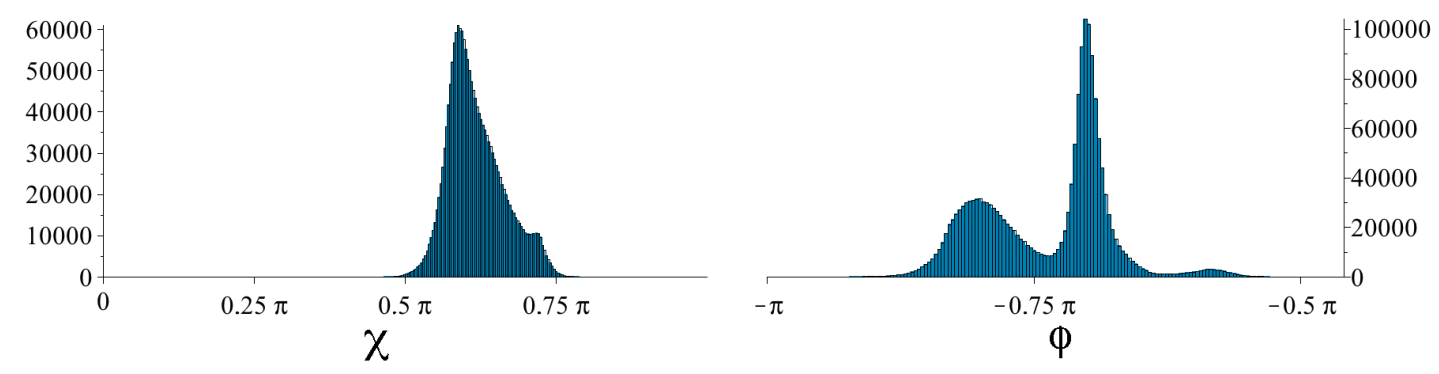

FIG. 11: Frenet frame histogram of the distribution of $(\chi, \varphi)$ angles displayed in Figure 10. for all $C_{\beta}$ in the PDB. The histogram shows how the directions are subject to only very small deviations around their average values.

Finally, we have found that in Bishop's parallel transport frame the direction of the $C_{\beta}$ carbon does not lead to such a regular structure formation as in the Frenet frame; See Figure 12 where we plot the statistical distributions of the vectors $\mathbf{c}$ in the Bishop's frames.

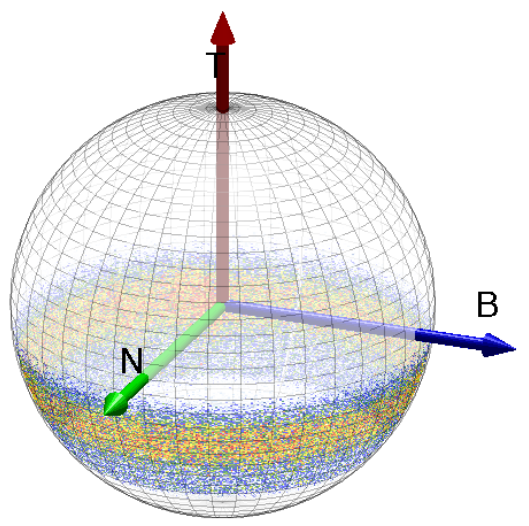

FIG. 12: The same as in Figure 10, but for all proteins in PDB using Bishop's parallel transport frame. In this frame the directions of the $C_{\beta}$ carbons are distributed in a longitudinally uniform manner inside a segment of the Kent sphere.

\section{V: CONCLUSIONS}

We have scrutinized the problem of frame determination along piecewise linear discrete curves, including those with inflection points. Our approach is based on the transfer matrix method that has been previously applied extensively 
to investigate discrete integrable systems and lattice field theories. The introduction of a transfer matrix enables us to describe a framing in a covariant manner, with different frames related to each other by $S O(2)$ gauge transformations that correspond to rotations in the normal planes of the curve. In particular our construction is not based on, and does not involve, any discretization of a continuum equation. Consequently we can effortlessly describe curves that become fractals in the limit where the lattice spacing a.k.a. the length of line segments vanishes. But we have also verified that if the continuum limit exists as a class $\mathcal{C}^{3}$ differentiable curve, we arrive at the generalized version of the continuum Frenet equation. Furthermore, the manifest covariance of our formalism under frame rotations enables us to investigate the framing of a physically determined discrete curve in a manner where the framing is based on, and captures the properties of the underlying physical system. Consequently we expect that our formalism has wide applications to the visualization of discrete curves and the determination of camera gaze positions in a variety of scenarios.

One notable outcome of our analysis is the identification of inflection points with the centers of loops, and the interpretation of loops as kink-solitons. In [28, 29] we have already applied this identification to develop an Ansatz based on (1), to succesfully describe the native folds of PDB proteins in terms of elementary functions.

As an example we have investigated the framing of folded proteins in the Protein Data Bank. In this case no valable continuum description exist, due to the fact that the universality class of folded proteins is characterized by the presence of a nontrivial Hausdorff dimension. Consequently any framing of folded proteins should be inherently discrete. In order to introduce a framing that directly relates to the physical properties of a folded protein, we have employed the relative orientation of the $C_{\beta}$ carbons in the amino acids with respect to the ensuing backbone central $C_{\alpha}$ carbons. We have statistically analyzed the relative orientation of these $C_{\beta}$ frames to the geometrically determined Frenet frames of the PDB proteins. We have found that the two framings are almost identical, they differ from each other only by a practically amino acid independent global frame rotation: For the $\alpha$-helices the nutation in the orientation of the $C_{\beta}$ carbons in the Frenet frame is very sharply concentrated around its statistically determined average direction. For $\beta$-strands the result is very similar, with only a relatively small increase in the amplitude of nutations. Finally, in the case of loops we find that the orientation of the $C_{\beta}$ carbons oscillates along a narrow circular arc that connects the $\alpha$-helices and $\beta$-carbons. In each case we have used the definition employed in the Protein Data Bank to identify the helix/loop class of the amino acid, and we note that the existing criteria for determining this class in the case of an amino acid that is located in the vicinity of the terminals of each structure is subject to interpretations. Consequently we propose that there are several border line cases that interfere destructively with the accuracy of our statistically determined results. We hope that our framing technique will eventually provide a refinement of the existing classification principles. The biophysical interpretation and biological relevance of our observations will be reported elsewhere.

\section{ACKNOWLEDGEMENTS}

We thank Maxim Chernodub for many valuable discussions and Jack Quine for communications.

[1] A.J. Hanson, Visualizing Quaternions, Morgan Kaufmann Elsevier (London) 2006

[2] J.B. Kuipers, Quaternions and Rotation Sequences: a Primer with Applications to Orbits, Aerospace, and Virtual Reality, Princeton University Press (Princeton) 1999

[3] R.L. Bishop, Amer. Math. Monthly 82 (1974) 246-251

[4] I. Montvay and G. Münster, Quantum Fields on a Lattice, Cambridge University Press (Cambridge) 1994

[5] U.H. Danielsson, M. Lundgren, A.J. Niemi, Phys. Rev. E 82 (2010) 021910-021914

[6] M. Spivak, A Comprehensive Introduction to Differential Geometry, Vo. 5 Publish or Perish (Houston) 1979

[7] A.J. Niemi, Phys. Rev. D67 (2003) 106004-106007

[8] H. Hasimoto, Journal of Fluid Mechanics (1972), 51: 477-485

[9] N. Manton and P. Sutcliffe, Topological Solitons (Cambridge University Press, Cambridge, 2004)

[10] H.M. Berman, K. Henrick, H. Nakamura, J.L. Markley, Nucl. Acids Res. 35, (Database issue) D301 (2007)

[11] C.J. McKnight, P.T. Matsudaira and P.S. Kim, Nat. Struct. Biol. 4, 180 (1997)

[12] J. Meng, D. Vardar, Y. Wang, H.C. Guo, J.F. Head and C.J. McKnight, Biochemistry 44, 11963 (2005)

[13] T.K. Chiu, J. Kubelka, R. Herbst-Irmer, W.A. Eaton, J. Hofrichter and D.R. Davies, Proc. Natl. Acad. Sci. U.S.A 102, $7517(2005)$

[14] L. Wickstrom, Y. Bi, V. Hornak, D.P Raleigh and C. Simmerling, Biochemistry 46, 3624 (2007)

[15] G. Jayachandran, V. Vishal and V.S. Pande, Journ. Chem. Phys. 124, 164902 (2006) 
[16] D.L. Ensign, P.M. Kasson and V.S. Pande, J. Mol. Biol. 374, 806 (2007)

[17] H. Lei and Y. Duan, J. Mol. Biol. 370, 196 (2007)

[18] P.L. Freddolino and K. Schulten, Biophys. Journ. 97, 2338 (2009)

[19] P.L. Freddolino, C.B. Harrison, Y. Liu and K. Schulten, Nature Physics 6 (2010) 751-758

[20] M. Chernodub, S. Hu and A.J. Niemi, Phys. Rev. E82 (2010) 011916-011920

[21] N. Molkenthin, S. Hu and A.J. Niemi, Physical Review Letters 106078102 (2011)

[22] S. Hu, A. Khorotkin, A.J. Niemi and X. Peng, E-print arXiv:1011.3181 [q-bio.BM]

[23] A.S. Davydov, Journ. Theor. Biology 38 (1973) 559

[24] Dill, K.A., Banu Ozkan, S., Shell, M.S. and Weikl, T.R., The Protein Folding Problem, Annual Review of Biophysics 37 289-316 (2008)

[25] G. Jayachandran, V. Vishal and V.S. Pane, J. Chem. Phys. 124164902 (2006)

[26] K. Huang, Lectures On Statistical Physics And Protein Folding (World Scientific Publishing Co. Pte. Ltd. Singapore, 2005)

[27] P.J. Flory, Statistical Mechanics of Chain Molecules (Wiley, New York, 1969)

[28] A.J. Niemi, Phys. Rev. D67 106004-106009 (2003)

[29] U.H. Danielsson, M. Lundgren and A.J. Niemi, Physical Review E82 021910 (2010) 